\section{Resumo}

O crescimento arbóreo é influenciado por fatores diversos intrínsecos e extrínsecos, variando não somente entre as espécies como também respondendo a variações ambientais e condições ecológicas, ou fatores de sítio. No entanto, o clima,

\title{
Influência do clima no crescimento diamétrico de Araucária angustifolia: revisão bibliográfica
}

\author{
Lais Martinkoski ${ }^{1}$ \\ Gabriel Felipe Voge ${ }^{2}$ \\ Sidne Osmar J adoski ${ }^{3}$
} mais especificamente a temperatura do ar e a precipitação se destacam como as variáveis climáticas que desempenham maior influência sobre o crescimento diamétrico de diversas espécies de clima temperado e subtropical, e muitas vezes, de clima tropical. Estudos dendrocronológicos com espécies nativas apresentam dados que geram informações relevantes para o manejo conservacionista e para a produção, além de potencial uso na reconstrução climática da região. A espécie Araucaria angustifolia é característica da Floresta Ombrófila Mista, apresentando caráter marcante nesta tipologia florestal e assim despertando interesse da pesquisa do ponto de vista conservacionista e potencial produtivo. O objetivo do presente trabalho foi estudar pesquisas referentes à dendrocronologia e influência de variáveis climáticas sobre o crescimento de $\mathrm{A}$. angustifolia em áreas de florestas nativas. Pode-se observar que de uma forma geral, o fator mais limitante em um momento mais crítico tende a expressar maior resposta, ou seja, precipitações e/ou temperaturas baixas coincidindo com o início da estação de crescimento tendem a formar anéis anuais mais estreitos, e assim um menor crescimento anual. De acordo com os trabalhos analisados pode-se destacar que quanto maior a precipitação e maior temperatura média durante a estação de crescimento, maiores anéis anuais tendem a ser encontrados, embora esta influência parece estar bastante atrelada a condições de sítio.

Palavras chaves: dendrocronologia, temperatura, precipitação

\section{Climate influence in diameter growth of Araucária angustifolia: literature review}

\begin{abstract}
The tree growth is influenced by several factors intrinsic and extrinsic, varying not only between species but between also responding to environmental changes and ecological conditions, or site factors. However, the weather, specifically the air temperature and precipitation stand out as the climatic variables that play a greater influence on the diameter growth of several species of temperate and subtropical climate, and often tropical climate. dendrochronological studies of native species present data to generate information relevant to conservation management and production, and potential use in climate reconstruction of the region. Araucaria angustifolia species is characteristic of the Araucaria Forest, with striking character in this forest type and thus arousing the interest of research conservationist standpoint and productive potential. The aim of this study was to research related to dendrochronology and influence of climatic variables on the growth of A. angustifolia in areas of native forests. It can be seen that in general, the most limiting factor in a most critical time is likely to express greater response, or precipitation and / or low temperatures coinciding with the beginning of the growing season tend to form more narrow annual rings, and thus a lower annual growth. According to the analyzed studies it can be noted that the greater precipitation and higher average temperature during the growing season, higher annual rings tend to be found, although this influence appears to be very tied to site conditions.
\end{abstract}

Key words: dendrochronology, temperature, precipitation

Received at: $14 / 02 / 15$
1 Msc. Produção Vegetal - Prof. Instituto Federal do Paraná/IFPR, Instituto Federal do Paraná/IFPR, campus Ivaiporã; lais.martinkoski@
ifpr.edu.br.
2 Aluno Departamento de Agronomia - Universidade Federal da Fronteira Sul (UFFS). Grupo de pesquisa em Manejo do solo, água e planta
em sistemas de produção, Email: gabrielfelipe02@hotmail.com.
3 Eng. Agrõnomo, Dr. Prof. Depto Agronomia - Universidade Estadual do Centro Oeste/UNICENTRO. Guarapuava-PR., Email: sjadoski@
unicentro.br.

Applied Research \& Agrotechnology 08 n2 may/aug. (2015)

Print-ISSN 1983-6325 (On line) e-ISSN 1984-7548 
Martinkoski et al. (2015)

\section{Influencia del clima en el crecimientodiamétricodeAraucária angustifolia:revisión de literatura}

\section{Resumen}

El crecimiento arbóreo es influenciado por varios factores intrínsecos y extrínsecos, que varían no sólo entre especies, sino también en responder a los cambios ambientales y las condiciones ecológicas, o factores de sitio. Sin embargo, el clima, especialmente la temperatura del aire y la precipitación se destacan como las variables climáticas que desempeñan una mayor influencia en el crecimiento del diámetro de varias especies de clima templado y subtropical, y muchas veces de clima tropical. Estudios dendrocronológicos con especies nativas presentan datos que generan informaciónes relevantes para el manejo conservacionista y para la producción, además de uso potencial en la reconstrucción del clima de la región. La especia Araucaria angustifolia es característica del bosque Ombrófila Mista, presentado carácter especifico en este tipo de bosque y así despertando el interés de la investigación del punto de vista conservacionista y del potencial productivo. El objetivo de este estudio fue estudiar las investigaciónes relacionadas con la dendrocronología y la influencia de las variables climáticas en el crecimiento de A. angustifolia en zonas de bosques nativos. Se puede observar que, en general, el factor más limitante en un tiempo más crítico tiende a expresar mayor respuesta, esto es, precipitación y / o bajas temperaturas coincidiendo con el inicio de la temporada de crecimiento tienden a formar anillos anuales más estrechas, y por lo tanto un menor crecimiento anual. De acuerdo con los estudios analizados se puede observar que cuanto mayor la precipitación y la temperatura media durante la estación de crecimiento, mayores anillos anuales tienden a encontrarse, a pesar de que esta influencia parece estar muy ligada a las condiciones del lugar.

Palabras clave: dendrocronología, temperatura, precipitación

\section{Introdução}

No Brasil, a expansão da atividade agropecuária sobre as áreas de floresta, aliada ao tradicional uso exploratório das espécies madeireiras nativas, veio a dificultar a manutenção e conservação de diversas espécies florestais nas diferentes tipologias florestais. A Floresta Ombrófila Mista (FOM) também conhecida como Floresta com Araucária, é característica com uma mistura de floras de origens temperada (austro-brasileira) e tropical (afro-brasileira) (MATTOS et al., 2010), a qual cobria originalmente uma área de $175 \mathrm{mil} \mathrm{km²}$, ocorrendo em especial na região sul do Brasil com algumas áreas ocasionais no estado de São Paulo, Minas Gerais e Rio de Janeiro (MEDEIROS, et al., 2005).

Atualmente, o cenário encontrado na FOM é de fragmentos de remanescentes florestais em sua maioria de tamanho reduzido e entre áreas antropizadas, no Paraná especificamente, essa floresta foi amplamente explorada por colonizadores europeus a partir do final do século XIX, impulsionando a economia do estado, mas levando a restar pouco mais de $1 \%$ de remanescentes em processos avançados de sucessão, os quais normalmente se encontram em unidades de conservação ou em áreas pouco transitáveis (SANQUETTA, 1995; STEPKA, 2012).
A Araucaria angustifolia (Bertol.) Kuntze conhecida popularmente como Pinheiro-do-Paraná é destacada como a árvore símbolo deste estado, sendo uma espécie bastante característica da FOM, presente de forma marcante em áreas com esta tipologia florestal. Segundo CARVALHO (2003) os indivíduos adultos de A. angustifolia são gimnospermas dióicas que têm entre 20 e $40 \mathrm{~m}$ de altura, tronco único e cilíndrico com diâmetro de 1 a $2 \mathrm{~m}$, copa umbeliforme e idades que variam geralmente de 150 a 250 anos, este autor ressalta ainda que esta espécie é uma heliófila, secundária longeva com características de pioneira, colonizando campos, inclusive em solos rasos.

O crescimento vegetal arbóreo é definido como o aumento das dimensões, as quais são principalmente o diâmetro, a altura, o volume, a biomassa e a área basal, sendo avaliados em um ou mais indivíduos de uma floresta sob um determinado período de tempo (PRODAN et al., 1997). O crescimento linear (elongação) de todas as partes da árvore é resultante da atividade do meristema primário. Já o crescimento em diâmetro (aumento da espessura) é proveniente da atividade do meristema secundário ou câmbio (HUSCH et al., 1982).

Conhecer a expressão do crescimento de árvores nativas e povoamentos florestais é fundamental para o planejamento da atividade 


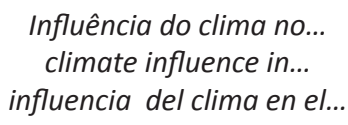

silvicultural (SCOLFORO, 1998). Assim, o estudo de incrementos diamétricos torna-se uma ferramenta indispensável, uma vez que apresenta o quanto uma floresta e/ou determinada espécie cresceu em diâmetro por um determinado período de tempo (MACHADO et al., 2014).

A presente revisão bibliográfica tem como objetivo reunir e analisar dados de pesquisas referentes à dendrocronologia e influência de variáveis climáticas sobre o crescimento de A. angustifolia em áreas de florestas nativas, de forma a contribuir para a discussão do tema.

Formação dos anéis de crescimento de dendrocronologia

ENCINAS et al. (2005) definem os anéis de crescimento como o resultado da deposição sucessiva de camadas de tecidos lenhosos no fuste, em razão da atividade cambial periódica, sendo que, a atividade do cambio ano a ano vai acrescentando camadas justapostas que irão formar o anel de crescimento por meio da estruturação do material lenhoso.

As árvores de clima temperado têm ritmos diferentes ao longo do ciclo de crescimento, sendo que, na primavera as plantas reiniciam a sua atividade cambial, após o inverno, que caracteriza um período de dormência, enquanto o lenho de primavera é caracterizado por paredes celulares delgadas e lúmens grandes, que vistos na seção transversal apresentam coloração clara (MEDEIROS, 2005; LAUW, 2011).

O lenho de outono ou de fim de estação, corresponde às células que vão diminuindo sua atividade vital à medida que o fim do período vegetativo se aproxima, tornando as paredes celulares mais espessas e os lúmens menores, deixando o conjunto com um aspecto mais escuro, sendo esta alternância de cores o que permite distinguir o crescimento relativo a cada ano (LAUW, 2011). MUNARETO (2007) destaca que esta diferenciação dos anéis, em geral, só é utilizada em espécies que possuem esta característica facilmente observável, contudo, essa diferenciação dos anéis pode ser influenciada por outras características como a idade, espaçamento e sítio.

A dendrocronologia é definida como o estudo dos anéis de crescimento das árvores, determinando assim a idade destas, suas bases teóricas vêm desde o século XVI, com Leonardo Da Vinci. No entanto, somente no século XX a dendrocronologia é solidificada como ciência, principalmente devido à influência de um astrônomo norte-americano chamado Andrew E. Douglass, que utilizou séries temporais de anéis de crescimento para relacioná-las com fenômenos climáticos e astronômicos, datando madeiras arqueológicas (FRITTS, 1976).

OLIVEIRA (2010) destaca que inúmeros fatores ambientais bióticos e abióticos regulam o crescimento das plantas, desta forma, anéis de crescimento são como "arquivos históricos" das condições ambientais uma vez que suas características também variam em função do ambiente, porém, a decodificação da informação existente nestes anéis apresenta-se de forma complexa devido à multiplicidade destes fatores ambientais, e principalmente da interação entre eles. Portanto, conhecer as respostas das plantas às condições ambientais é fundamental para interpretar os padrões temporais de variação dos anéis de crescimento.

A dendrocronologia baseia-se sob alguns princípios (FRITTS, 1976), sendo estes:

a)Princípio da uniformidade: os mesmos processos físicos e biológicos que afetam os padrões de crescimento das árvores na atualidade, afetaram no passado;

b)Princípio dos fatores limitantes: o crescimento dos anéis de uma árvore é mais influenciado pelo fator ambiental mais limitante, por exemplo, a precipitação em regiões secas, ou ainda a temperatura em maiores latitudes;

c) Princípio do crescimento agregado da árvore: a origem da variação pode ser decomposta por um conjunto de fatores ambientais ou bióticos;

d)Princípio da amplitude ecológica: as espécies são mais sensíveis a alterações climáticas quanto mais perto estiverem dos limites de altitude e latitude de sua área de habitat;

e)Princípio da seleção do local: os locais mais favoráveis para a dendrocronologia podem ser identificados e selecionados com base em critérios que produzirão séries de anéis de crescimento sensíveis à variável ambiental em estudo;

f)Princípio da repetição: a obtenção de mais de um raio por árvore e mais de uma árvore por local diminui a influência de fatores ambientais que não estão sendo estudado interferirem nos resultados;

g)Princípio da datação cruzada: é o princípio fundamental da dendrocronologia, nele, é definido que a correspondência de padrões da largura dos anéis entre diversas séries de anéis, este permite a identificação do ano exato em que cada anel da árvore foi formado. É realizado considerando dois 
Martinkoski et al. (2015)

raios por árvore e a sincronia das diferentes séries de cada árvore. A datação cruzada é realizada também entre as diferentes árvores, a fim de se verificar picos de crescimento comuns, e assim caracterizar anos típicos, ou seja, anos em que as árvores tiveram crescimento similar.

Segundo FINGER (1992) durante a contagem dos anéis é necessário cuidado relativo à existência de falsos anéis, que poderão induzir a atribuição de mais um ano, tal distinção é feita verificando-se a continuidade do anel em uma seção transversal, ou, no caso de amostras não-destrutivas, pela coleta de mais de um rolo de incremento por árvore.

A forma como essas informações são usadas tratam do estudo dos problemas do presente por meio dos anéis produzidos em anos anteriores, por meio de fatores climáticos ou ecológicos que interferiram no crescimento, assim, é possível prever mudanças no ambiente local da avaliação e alterar o manejo adotado (ABRAMS e COPENHEAVER, 1999).

Fatores que afetam o crescimento de espécie arbóreas

O crescimento de uma árvore é definido como o aumento gradativo de tamanho de raízes, copa e tronco, em resposta não somente aos processos naturais de vida, como também a estímulos ambientais (ZANON E FINGER, 2010). Portanto, o crescimento das árvores depende dos fatores ambientais como luz, temperatura e umidade; espaços físicos; edáficos; topográficos; tamanho e constituição genética das árvores; histórico de desenvolvimento da floresta; além de fatores relacionados à competição como, por exemplo, a densidade do povoamento (POORTER e BANGERS, 1993; ANDRADE, et al., 2007).

De acordo com HIGUSCHI et al. (2003) o crescimento diamétrico das árvores varia entre e dentro das espécies em função não só da idade, como das estações do ano e das condições climáticas. Pesquisas evidenciam que o crescimento em algumas espécies florestais tropicais e subtropicais se apresenta de forma intermitente, afetando os processos fisiológicos das árvores, o que reflete na atividade cambial.

Na FOM, em A. angustifolia, ZANON e FINGER (2010), concluíram que o crescimento diamétrico se inicia em setembro, tendo seu pico em janeiro e decrescendo a partir de março e estagnando em julho e agosto, ressaltando que o mês do pico de crescimento tende a variar em função das condições climáticas de cada ano. Neste contexto, MARIA (2002) verificou que a redução da taxa de incremento ocorria geralmente a partir de março nas espécies Cedrela fissilis, Tabebuia serratifolia, Cariniana estrellensis, Copaifera langsdorffi, Balfourodendrum riedelianum e Centrolibium tomentosum, atribuindo essa redução no crescimento à diminuição ou cessamento da atividade cambial decorrente do início do déficit hídrico, em conjunto com as temperaturas mais baixas que antecediam o início do inverno. Sendo assim, verifica-se que os estudos na área utilizam principalmente dados referentes a temperatura média do ar e a precipitação pluviométrica nos locais onde as espécies arbóreas são estudadas.

\section{Precipitação pluviométrica}

A água está relacionada ao crescimento devido a sua participação em todos os processos fisiológicos e bioquímicos, sendo a principal constituinte dos tecidos vegetais, responsável pela turgescência celular, reacionante em processos hidrolíticos e atuando como meio de dissolução de sais e outras substâncias, criando um sistema dissolvente contínuo no interior da planta (CAMPOS, 1970). Este mesmo autor destaca ainda que, o crescimento não é diretamente controlado pelo teor de umidade do solo, mas sim, pelo balanço de água na planta, o qual é afetado do teor de umidade no solo e na atmosfera, no entanto, se houver déficit de água interno na planta, ocorre uma redução no crescimento como resposta ao fechamento estomático e a diminuição na divisão e alongamento celular.

Em regiões tropicais, a redução da atividade cambial se deve a diminuição da precipitação, ou ainda a inundação, no caso de áreas alagáveis. Em 1931 Berlege realizou a primeira cronologia de anéis anuais com mais de 400 anos nos trópicos, utilizando árvores de Tectona grandis L. f. (Verbenaceae), onde este autor conseguiu relacionar o regime de precipitação na Indonésia, com os índices de incremento da espécie, destaca-se que antes deste fato ocorrer, este tipo de estudo limitava-se a regiões temperadas como Europa e EUA (ROSA, 2008).

A variação do regime de chuvas foi o fator determinante na periodicidade da formação do xilema em diversos estudos. SOUZA et al. (2006) destacam que os efeitos diretos e indiretos da deficiência de água no crescimento das árvores irão influenciar na relação da disponibilidade hídrica do solo e na produtividade florestal. JACOBY (1989) estudando o crescimento radial da teca (Tectona 


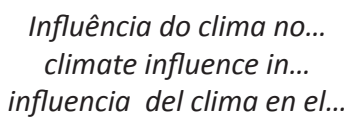

grandis) na Índia e em Java pode concluir que este foi influenciado pela quantidade de chuva durante a estação seca em ambas as localidades.

OLIVEIRA et al. (2011) estudando a densidade dos anéis de crescimento em Eucalyptus grandis, verificaram que quanto maiores os valores de precipitação, maior o crescimento anual encontrado. MATTOS et al. (2004), encontraram correlação positiva entre o incremento radial e a precipitação pluviométrica para discos de Tabebuia heptaphylla proveniente do Pantanal Mato-Grossense. BRIENEN e ZUIEDEMA (2005) e WORBES (1999) também encontraram relação positiva e elevada entre os anéis de crescimento e precipitação em certos períodos do ano para espécies tropicais da Amazônia.

Com relação à $A$. angustifolia, Mattos et al. (2007b) concluíram que o crescimento de uma forma geral, foi pouco influenciado pelas condições climáticas locais, no entanto, houve uma redução do crescimento após um ano em que houve baixa precipitação e umidade relativa durante o início da primavera. ZANON e FINGER (2010) encontraram resultados que evidenciaram a precipitação como sendo um fator que influiu positivamente no incremento médio anual desta espécie, sendo que, nos meses em que ocorreu uma quantidade de chuvas abaixo da média durante o final do verão, o crescimento foi bastante reduzido, segundo estes autores, estas respostas ocorreram, pois à medida que o solo vai secando nos meses de menor pluviosidade durante a estação de crescimento, todos os processos fisiológicos vão sendo restringidos.

STEPKA (2012) verificou que houve correlação significativa entre a precipitação e o incremento de araucária em uma floresta madura localizada em São Francisco de Paula-RS, porém, não encontrou este efeito de forma significativa nas demais áreas sob abrangência da FOM avaliadas em seu estudo. No Paraná, FIGUEIREDO FILHO et al. (2008) encontraram correlações do crescimento com a precipitação em algumas espécies, incluindo $A$. angustifolia. MATTOS et al. (2007b) observaram efeito de diminuição do crescimento em anos de evento "El nino" mais acentuado, ou seja, estiagens na primavera-verão, em especial no início da primavera, gerando anéis mais estreitos.

No final da década de 90, o "El niño" foi considerado o mais importante evento meteorológico do século, causando anomalias na distribuição de chuvas em todas as regiões do país e maiores efeitos em diminuição de chuvas nas regiões sudeste e sul (RAO et al., 2001).
Corroborando com este resultado, CAUM (2013) estudando louro-pardo e CALLADO e GUIMARÃES (2010) estudando Schizolobium parahyba na região sudeste, observaram estreitamento dos anéis e assim diminuição no crescimento, como resultado deste evento de diminuição drástica de chuvas.

\section{Temperatura do ar}

CAMPOS (1970) destaca que a radiação solar constitui a fonte primária de energia do processo fotossintético, sendo assim, o efeito da radiação sobre o crescimento das plantas pode ser manifestado de várias formas, seja através da intensidade, qualidade ou duração. Como esta radiação é transformada em calor, a temperatura pode tornar-se um fator climático decisivo no crescimento das árvores, em especial em clima temperado. Segundo este mesmo autor, a quantidade de radiação incidente é variável em função da altitude, latitude e estação do ano e assim, consequentemente, a temperatura também sofre oscilações segundo estas condições.

Um fator climático pode afetar processos fisiológicos e assim influenciar no crescimento, neste contexto, a temperatura atua afetando o crescimento devido a esta influenciar na parada e na retomada da atividade cambial, podendo estes ocorrer em épocas do ano distintas entre diferentes anos e, assim, formando-se anéis com espessuras distintas, logo, a temperatura estaria diretamente relacionada com a espessura dos anéis (LAUW, 2011).

Temperaturas elevadas durante a estação de crescimento também são consideradas fatores limitantes, uma vez que estas podem limitar a produção de enzimas e hormônios que são necessários para que determinados processos fisiológicos ocorram, além de, acentuar a perda de água pelas plantas, em uma época em que muitas vezes, esta já é escassa, afetando assim o crescimento (FRITTS, 1976). Ainda segundo este autor, temperaturas baixas extremas durante o inverno, mesmo sob um prevalecimento de temperaturas abaixo de zero durante um longo período, podem não afetar processos fisiológicos, não havendo relação deste fator com a espessura dos anéis, no entanto, se houverem temperaturas mais elevadas durante o inverno poderá haver um descongelamento dos tecidos ocasionando retomada da atividade fisiológica, o que gera uma correlação entre as temperaturas de inverno e a espessura dos anéis de crescimento.

Em A. angustifolia, ZANON e FINGER (2010) verificaram que durante a estação de crescimento

Applied Research \& Agrotechnology v8 n2 may/aug. (2015)

Print-ISSN 1983-6325 (On line) e-ISSN 1984-7548 
Martinkoski et al. (2015)

o aumento da temperatura correspondia ao maior crescimento, o qual era cessado ou reduzido sob temperaturas mais baixas, sendo assim, os maiores incrementos em diâmetro das árvores ocorriam nos meses de maiores temperaturas médias mensais, enquanto nos meses de inverno, ou seja, fora da estação de crescimento, a redução da temperatura pareceu ser o elemento causador da interrupção do crescimento. Reforçando este resultado, OLIVEIRA et al. (2010) destaca que a sazonalidade das temperaturas encontrada em regiões subtropicais do Brasil explicou a variação encontrada nos anéis de crescimento de A. angustifolia.

No Paraná, em São João do Triunfo, FIGUEIREDO FILHO et al. (2008) ao analisarem o incremento desta espécie por meio de cintas dendrométricas durante sete anos, verificaram uma elevada correlação entre incremento e temperatura média do ar. STEPKA (2012) estudando o efeito da temperatura média do ar sobre o crescimento através de dendrocronologia de indivíduos adultos desta espécie em diferentes regiões da FOM, verificou que o efeito variou conforme a região, sendo mais pronunciado em São Francisco de Paula-RS e São João do Triunfo-PR do que nas demais regiões avaliadas.

MATTOS et al. (2007a) destacou que apesar de as condições climáticas locais não terem influenciado de forma pronunciada no crescimento de seis espécies da FOM, entre elas A. angustifolia, a ocorrência de um ano de baixa precipitação na estação de crescimento seguido de um período de baixas temperaturas no inverno seguinte acarretou em uma diminuição no crescimento das árvores no mesmo ano ou no ano seguinte.

\section{Conclusões}

O crescimento diamétrico de A. angustifolia depende de diversos fatores, destacando-se o clima e as condições de sítio, além da idade e posição fitossociológica, de modo que a maior influência climática pode ocorrer devido a precipitação pluviométrica ou então à temperatura média do ar, dependendo das demais condições citadas, ou seja, dependendo do local.

De uma forma geral, o fator mais limitante em um momento mais crítico tende a expressar maior resposta, ou seja, precipitações e/ou temperaturas baixas coincidindo com o início da estação de crescimento tendem a formar anéis anuais mais estreitos, e assim um menor crescimento anual. De acordo com os trabalhos analisados pode-se destacar que quanto maior a precipitação e menor a oscilação da temperatura média durante a estação de crescimento, maiores valores em incremento anual tendem a ser encontrados.

\section{Referências}

ABRAMS, M. D.; COPENHEAVER, C. A. Temporal variation in species recruitment and dendroecology of an old growth white oak forest in the Virginia Piedmont, USA. Forest Ecology and Management, v.124, p. 275-284, 1999.

BRIENEN, R. J. W.; ZUIDEMA, P. A. Relating tree growth to rainfall in Bolivian rain forests: a test for six species using tree ring analysis. Oecologia, v. 146, n.1, p. 1-12, 2005.

CALLADO, C. H.; GUIMARÃES, R. C. Estudo dos anéis de crescimento de Schizolobium parahyba (Leguminosae: Caesalpinioideae) após episódio de mortalidade em Ilha Grande, Rio de Janeiro. Revista Brasileira de Botânica. v. 33, n.1, p. 85-91, 2010.

CAMPOS, J. C. C. Principais fatores do meio que afetam o crescimento das árvores. Revista Floresta, v. 2, n. 3, p. 45-52, 1970.

CARVALHO, P. E. R. Espécies arbóreas brasileiras. V. 1. Brasília, DF: Embrapa Informações Tecnológicas; Colombo, PR: Embrapa Florestas, 2003. 1039 p.

CAUM, C. Anatomia comparada da madeira de Cordia trichotoma (Vell.) Arráb. ex Steud. (BORAGINACEAE) proveniente de sementes de duas procedências e análise dos anéis do crescimento. 102f. Dissertação (Mestrado em Ciências Florestais) - Universidade Estadual Paulista “Júlio de Mesquita Filho” - UNESP, Botucatu, 2013. 


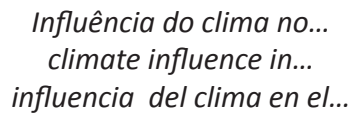

ENCINAS, J. I.; SILVA, G. F.; PINTO, J. R. R. Idade e crescimento das árvores. Comunicações Técnicas Florestais, Brasília, v. 7, n. 1, p. 1 - 43, 2005.

FIGUEIREDO FILHO A.; RODE, R.; FIGUEIREDO, D. J.; MACHADO, S. A. Seasonal diameter increment for 7 species from an Ombrophyllous Mixed Forest, southern state of Paraná, Brazil. Floresta, v. 38, n.3, p. 527-543, 2008.

FINGER, C. A. G. Fundamentos de biometria florestal. 1 ed. Santa Maria: UFSM/CEPEF/FATEC, 1992. 269 p. FRITTS, H.C. Tree Rings and Climate. London: Academic Press, 1976. 567 p.

HIGUCHI, N. Chambers, J.Q.; Silva, R.P. da; Miranda, E.V. de; Santos, J. dos; Iida, S.; Pinto, A.C.M.; Rocha, R.M.; Souza, C.A.S. de. Uso de bandas metálicas e dendrômetros automáticos para a definição do padrão de crescimento individual das principais espécies arbóreas da floresta primária da região de Manaus, Amazonas, Brasil. In: Projeto Jacarandá Fase II: Pesquisas Florestais na Amazônia Central, Manaus: INPE, p. 55-68, 2003.

HUSCH, B.; MILLER, C. I.; BEERS, T. W. Forest mensuration. 3ed. New York: John Wiley e Sons, 1982. 402p. JACOBY, G. G. Overview of tree ring analyses in tropical regions. IAWA Journal, Leiden, v.10, n.2, p. 99-108, 1989.

LAUW, A. M. R. F. Estudo de anéis anuais de crescimento de árvores como indicadores climáticos em Portugal. 2011. 84 f. Dissertação (Mestrado em Engenharia Florestal e dos Recursos Naturais) - Universidade técnica de Lisboa, Lisboa, 2011.

MACHADO, S. de A.; ZAMIN, N. T.; NASCIMENTO, R. G. M.; SANTOS, A. A. P. Efeito de Variáveis Climáticas no Crescimento Mensal de Pinus taeda e Araucaria angustifolia em Fase Juvenil. Floresta e Ambiente, Seropédica, v. 21, n.2, p.170-181, 2014.

MATTOS, P. P.; SEITZ, R. A.; SALIS, S. M. Potencial dendroecológico de Tabebuia heptaphylla (Vell.) Toledo. Boletim de Pesquisa Florestal, Colombo, n. 48, p. 93-103, 2004.

MATTOS, P. P.; SANTOS, A. T.; RIVERA, H.; OLIVEIRA, Y, M. M.; ROSOT, M. A. D.; GARRASTAZU, M. C. Crescimento de Araucaria angustifolia na Reserva Florestal

Embrapa/Epagri, Caçador, SC. Pesquisa Florestal Brasileira, Colombo, n. 55, p. 107-114, 2007 a.

MATTOS, P. P. de; SANTOS, A. T. dos; OLIVEIRA, Y. M. M. de; ROSOT, M. A. D.

Dendrocronologia de espécies da Floresta Ombrófila Mista do Município de Candói, PR. Pesquisa Florestal Brasileira, Colombo, v. 29, n.54, p.153-156, 2007 b.

MATTOS, P. P. de; OLIVEIRA, M. F.; AGUSTINI, A. A.; BRAZ, E. M.; RIVEIRA, H.; OLIVEIRA, Y. M. M. de; ROSOT, M. A. D.; GARRASTAZU, M. C. Aceleração do crescimento em diâmetro de espécies da Floresta Ombrófila Mista nos últimos 90 anos. Pesquisa Florestal Brasileira, Colombo, v.30, n.64, p.319-326, 2010.

MARIA, V. R. B. Estudo da periodicidade do crescimento, fenologia e relação com a atividade cambial de espécies arbóreas tropicais de florestas estacionais semidecíduas. 126 f. Dissertação (Mestrado em Recursos Florestais) - Universidade de São Paulo, Piracicaba, 2002.

MEDEIROS, J. D.; SAVI, M.; BRITO, B. F. A. de. Seleção de áreas para criação de unidades de conservação na Floresta Ombrófila Mista. Biotemas, Florianópolis, SC, v. 18, n. 2, p. 33-50, 2005.

MUNARETO, F. F. Dendroclimatologia de quarto espécies florestais nativas com potencial silvicultural e econômico. 2007. 62 f. Dissertação (Mestrado em Engenharia Florestal) - Universidade Federal de Santa Maria, Santa Maria, 2007.

OLIVEIRA, J. M.; ROIG, F. A.; PILLAR, V. D. Climatic signals in tree-rings of Araucaria angustifolia in the southern Brazilian highlands. Austral Ecology, v. 35, p. 134-147, 2010.

OLIVEIRA, B. R. U.; LATORRACA, J. V. de F.; FILHO, M. A.; GARCIA, R. A.; CARVALHO, A. M. de. Correlações dendroclimatológicas do Eucalyptus grandis Hill ex Maiden na região de Rio Claro, RJ. Ciência Florestal, Santa Maria, v. 21, n. 3, p. 499-508, 2011. 
Martinkoski et al. (2015)

RAO, V.B.; SANTO, C.E.; FRANCHITO, S.H.A diagnosis of rainfall over South America during 1997/98 El Niño event. Part I: validation of NCEP - NCAR reanalysis of rainfall data. Journal of Climate, v. 15, n. 1, p. 502-511, 2001.

ROSA, S. A. Modelos de crescimento de quatro espécies madeireiras de floresta de várzea da Amazônia central por meio de métodos dendrocronológicos. 77 f. Dissertação (Mestrado em Ciências Biológicas) Universidade Federal do Amazonas, Manaus, 2008.

PRODAN, M.; PETERS, R.; COX, F.; REAL, P. Mensura Forestal. San Jose, C.R.: Deutsche Gesellschaft fur Technische Zusammenarbeit (GTZ) GmbH: Instituto Interamericano de Cooperacion para la Agricultura (IICA), 1997. $561 \mathrm{p}$.

SANQUETTA, C. R. Análise da estrutura vertical de florestas através do diagrama hm. Ciência Florestal, Santa Maria. v. 5, n. 1, p. 55-67, 1995.

SCOLFORO, J. R. Biometria Florestal: Modelagem do crescimento e da produção de florestas plantadas e nativas. Lavras: UFLA/FAEPE; 1998. 441 p.

SOUZA, M. J. H.; RIBEIRO, A.; LEITE, H. G.; LEITE, F. P.; MINUZZI, R. B. Disponibilidade hídrica do solo e produtividade do eucalipto em três regiões da Bacia do Rio Doce. Revista Ârvore, Viçosa, v. 30, n.3, p. 399-410, 2006.

STEPKA, T. F. Modelagem do crescimento e dendrocronologia em árvores nativas de Araucaria angustifolia, Cedrela fissilis e Ocotea porosa no sul do Brasil. $186 \mathrm{f}$. Tese (Doutorado em Engenharia Florestal) - Setor de Ciências Agrárias, Universidade Federal do Paraná, Curitiba. 2012.

WORBES, M. Annual growth rings, rainfall-dependent growth and long-term growth patterns of tropical trees from the Caparo Forest Reserve in Venezuela. Journal of Ecology, v. 87, p. 391- 403, 1999.

ZANON, M. L. B.; FINGER, C. G. Relação de variáveis meteorológicas com o crescimento das árvores de Araucaria angustifolia (Bertol.) Kuntze em povoamentos implantados. Ciência Florestal, v. 20, n. 3, p. 467-476, 2010. 